\title{
BEAM DYNAMICS ISSUES OF HIGH-LUMINOSITY ASYMMETRIC COLLIDER RINGS
}

\author{
A.M. Sessler
}

\section{Abstract}

The beam dynamics issues presented by a high-luminosity asymmetric electron collider ring (such as is required for a $B$ meson Factory) are described. Attention is focused on lattice aspects, on single-beam effects, and on beam-beam interaction effects. The overall conclusion is that a facility with a beam of (about) $3 \mathrm{GeV}$ in one ring and a beam of (about) 9 GeV in a second ring having a luminosity of between $10^{33}$ and $10^{34} \mathrm{~cm}^{-2} \mathrm{~s}^{-1}$ is a feasible concept.

\section{Introduction}

The desire to study, in great detail, the $B-\bar{B}$ system and, in particular, to study the CP violation in that system, has motivated the development of very high-luminosity asymmetric collider rings [1]. The development of such a collider presents new challenges to accelerator physicists, and in order to explore and assess the beam dynamics issues that this quest raises, a workshop on the subject was called by the Lawrence Berkeley Laboratory (LBL) and the Stanford Linear Accelerator Center (SLAC) in February of this year [2].

The physics to be done at a $B$ Factory requires an integrated luminosity of more than $30 \mathrm{fb}^{-1} /$ year [1] and [3]. This is equivalent to a collider delivering a luminosity of at least $3 \times 10^{33} \mathrm{~cm}^{-2} \mathrm{~s}^{-1}$ for a third of each year $\left(10^{7} \mathrm{~s}\right)$. The required luminosity is larger than the present performance of colliders, in the same energy range, by a factor of at least 30 . In addition, the collider must have a c.m. energy of 10 to $11 \mathrm{GeV}$ with beam energy ratios of up to 5 to 1 (if the collider is symmetric in energy, then the luminosity required is larger than that given above by an addilional factor of $\sim 5$ ). From the machine physicist's point of view the extrapolation in luminosity is much more of a challenge than the extrapolation from symmetric colliders to asymmetric ones.

In this article, we shall draw heavily upon the workshop. On the very closing day of the workshop, a small group of physicists gathered together and attempted to summarize the conclusions in a succinct form. The first section of their summary is presented in a companion note following the present article.

Beam dynamics issues may conveniently be broken into three categories. The first is that having to do wich "single particle" phenomena. Under this comes the design of a proper focusing lattice, RF acceleration, injection, extraction, radiation damping, quantum fluctuations, etc.

The second category consists of single-beam phenomena arising from the many-body aspects of a beam. Within this category are conventional "space charge phenomena" (negligibly small at relativistic energies), and also rather sophisticated phenomena such as intrabeam scattering, synchro-betatron mode coupling, and single and multi-bunch coherent instabilities.

The third category consists of those phenomena that result from the interaction between beams where the non-linear forces are the primary source of concern.

In this paper we shall consider the beam dynamics of $B$ Factories by discussing, in turn, single-particle phenomena, single-beam phenomena, and beam-beam phenomena. We shall not be concerned with various "practical" issues such as injection, $e^{+}$production, vacuum, etc. They are, of course, important. We do note that the large luminosity implies a short beam lifetime and hence a dedicated injector and (probably) the ability to take data while "topping off" the beam. We start, first, with some general considerations.

\section{General considerations}

Some of the elements that must be considered by the machine physicist are shown in fig. 1. Of course, we are not starting from scratch; circular colliders have been built, and carefully studied, for two decades. It is quite appropriate to ask in that context what must be done differently from that which has been done in existing colliders in order to achieve the performance specifications of a $B$ Factory. In fact, this mode of reasoning is very simple, and almost unique in its results, so that all of the various proposals for $B$ Factories (sect. 6) are quite similar in general nature.

The reasoning begins as follows: the beam-beam interaction puts a limit on the luminosity created by one bunch (meeting one bunch of the other beam) which we presently do not know how to exceed. Wc can make this limit as large as possible by focusing the beams to a very small size at the crossing point (low $\left.\beta^{*}\right)$. But to get the required very large luminosity with a reasonable beam emittance will still require many bunches in each beam. Because of the many potential near-crossings (even with the separation that can be achieved electrostatically) the collider needs to have two rings. 


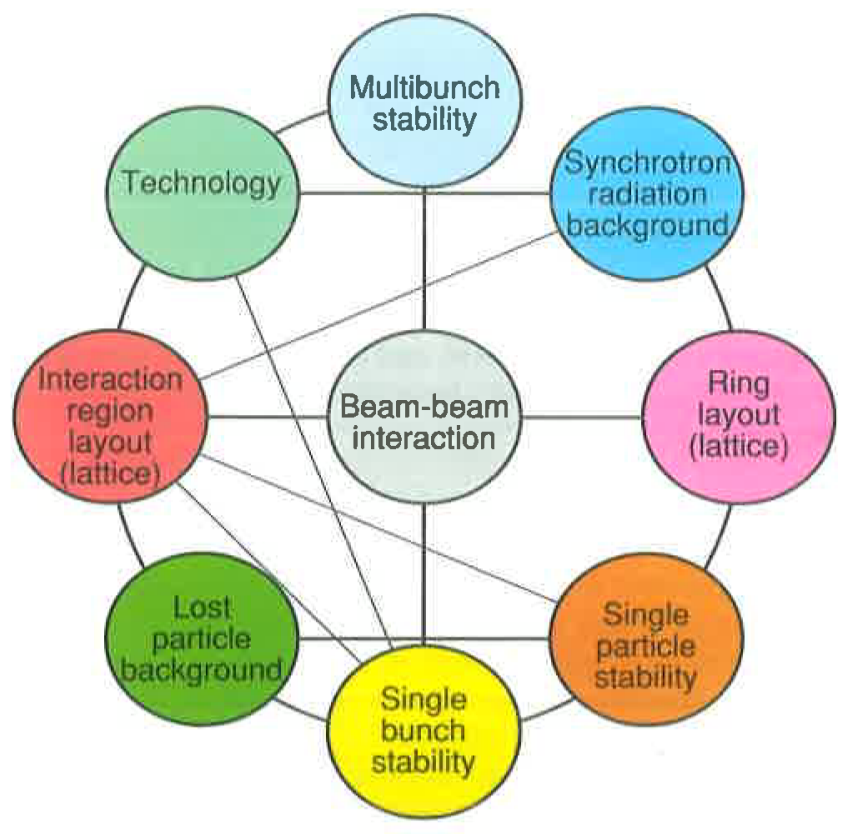

FIGURE 1

Diagram showing the various phenomena and their major interconnections that must be considered in designing a high-luminosity circular collider. Of course, at some level, every circle is connected to every other circle. Technical feasibility is a dominant consideration and is included, really, at all levels by "knowing what can and cannot be done". Notice that cost, which in the last analysis is the determining factor, is completely left out of the diagram (figure due to Maury Tigner).

What are the consequences of this direction for the design? The first thing with which we must be concerned is multi-bunch effects, and we shall discuss this more in sect. 4. Suffice it to note here that, due to the large current in each ring, there are severe multi-bunch instabilities and they must be handled by strong feedback systems. Even then it is critical to reduce their growth rates in the first place by proper design of RF cavities with reduced higher-order mode response. A second major consequence of the design is that the bunches must be separated rather close to the interaction point (because unwanted crossings must be avoided and the many bunches are close together). If the collisions are head-on - and experience suggests that the deleterious aspects of the beam-beam interaction are greatly enhanced if the crossing is not head-on - then powerful magnets are required near the crossing point and these produce synchrotron radiation background from which the detector must be shielded. We shall discuss this in sect. 3. Alternatively, the crossing could be at an angle, but appear as if it is head-on; this approach would employ the suggestion by Bob Palmer of "crab crossing" (described below) [4]. This scheme, which has not yet been studied very extensively, does not require the use of separa- tion magnets and consequently is good from a masking point of view, but requires strong crab RF cavities near the interaction point. The technical feasibility of this scheme is unknown at this time. Being able to focus both a high-energy beam (HEB) and a low-energy beam (LEB) by a common set of magnets in the interaction region (IR) implies a novel and challenging feature of asymmetric machines. A third consequence of the design is that the very low $\beta^{*}$ implies a concomitant need for very short bunches and, hence, a very powerful RF system. It is clear from both the above that the RF system must be of special design that can deliver a large amount of power and voltage to the beam with a minimum number of cavities.

There are other consequences of our design direction, and some of them will be touched upon below. Much more can be found in the various design study documents being produced in the laboratories mentioned in sect. 6 , but the major consequences are as listed above and depicted in fig. 2 . One cannot help but notice from fig. 2 and fig. 1, where all the issues seem to converge on the IR layout circle, that the design of the interaction region optics plays a central and crucial role in any highluminosity collider design. No other aspect is as intricately connected to all others as is the interaction region.

\section{Lattices}

Perhaps one should start with consideration of the beambeam interaction, for that is central to a $B$ Factory design. Fortunately, however, the consequences of this subject can be summarized very succinctly, and that allows us to proceed in the logical order of designing a collider for single-particle effects and then, subsequently, concerning ourselves with many-particle phenomena. Of course, life is not that simple and there must be continual interchange between the experts in lattices and in many-body phenomena.

The physics of single-particle behaviour in colliders has been set out in the classic work by Matt Sands [5]. Although that work is twenty years old, it includes just about everything one needs to know to design a collider. We shall not go through considerations that are well known, such as betatron tune, chromaticity, dispersion, radiation damping times and emittance, although all of these are needed to design a collider (for example, we shall not comment upon the required beam emittance which is low, but in the range that has already been achieved). Rather, we shall comment, only in a very general way, upon the novel features that enter into $B$ Factory design.

Perhaps the central complicating feature of the design is that there must be two rings (not completely new ground; think of the ISR or HERA). Thus, the interaction region, with its separation of particles and its production of a very low $\beta^{*}$ at the crossing point, is the most difficult part of the design. Of course, one must be concerned with chromaticity corrections, making straight sections in which wigglers can be inserted to produce and control low 


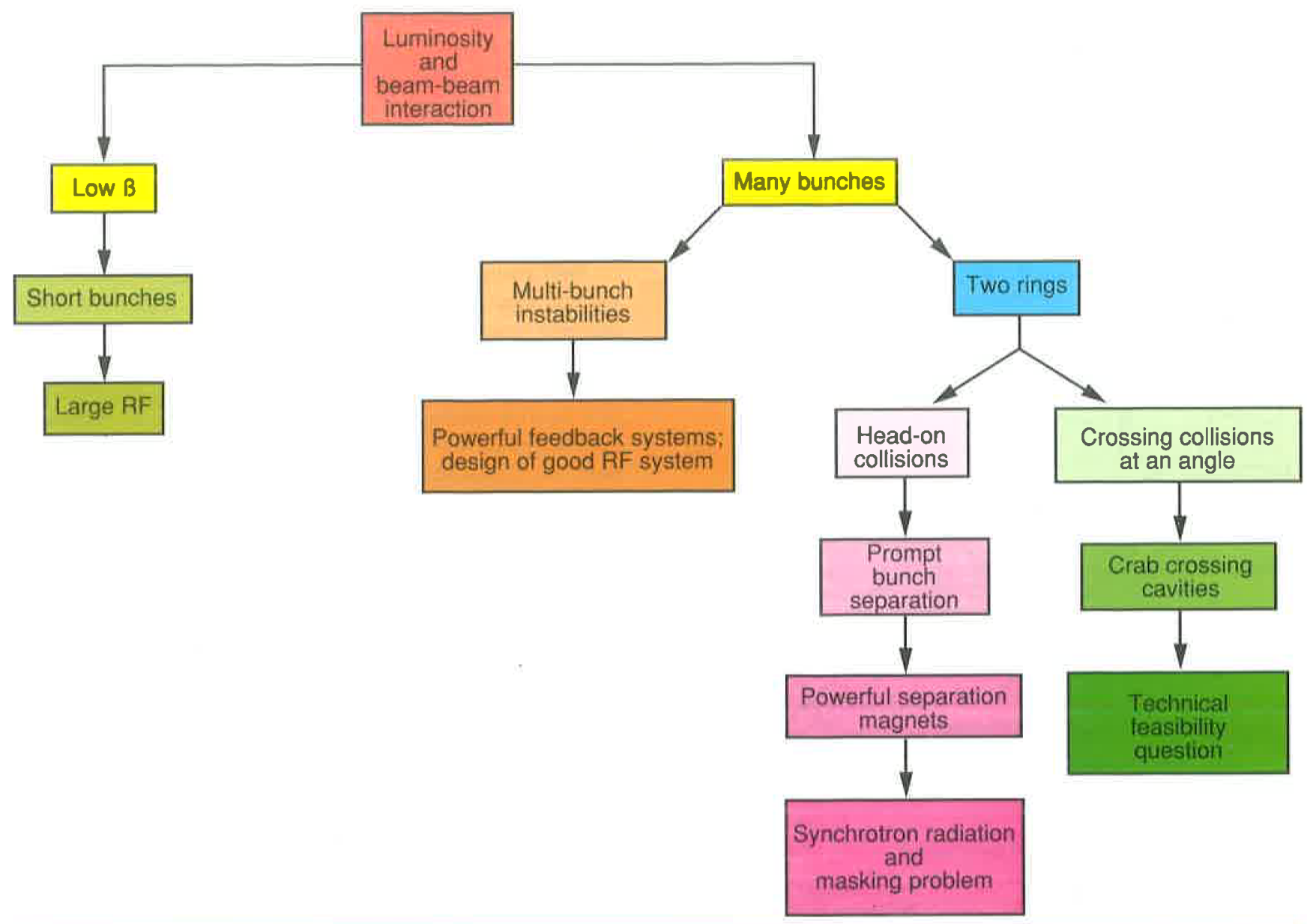

\section{FICIRE 2}

The logical steps that one takes in designing a high-luminosity collider. Some explanation and futher analysis are given in sect. 2 of the paper along with further details in sects 3,4 and 5 .

beam emittance and short damping times, and the myriad of other things that go into a lattice design. But the main complication comes about with designing the interaction region.

The difficulty is in the combined aspect of producing a low $\beta^{*}$ and separating the beams, while at the same time not producing too much synchrotron radiation very near the interaction point. The low $\beta^{*}$ can be produced by powerful focusing quadrupoles, but as the beams are separated, the one that is offcentre in the quadrupole feels a large field and consequently bends and radiates (the one on-centre also radiates, but only because of its finite size). In an obvious way, any dipole magnets that are employed to separate the unequal energy beams also produce synchrotron radiation from both beams. Rather strong magnets are needed to get prompt separation (as one moves away from the interaction point) because of the close bunch spacing.
A number of different suggestions have been made, and presently are being explored, for the interaction region geometry (sect. 6). In fig. 3 we have indicated the essential elements of two of these suggestions. As of this writing, no completely acceptable solution has been produced, although there is no reason to believe that one cannot be achieved. Of course, there needs to be considerable attention to the quality and nature of the required synchrotron radiation masks and the sensitivity of the detector to radiation. In addition to synchrotron radiation, there is the background from lost particles which is strongly affected by the beam-beam interaction that is the primary mechanism for putting particles into the tail of beam distributions.

One issue in the design is whether the beam is flat (aspect ratio of say 40 to 1) or round. It is unclear how much one gains in the beam-beam interaction with round beams (as discussed in 

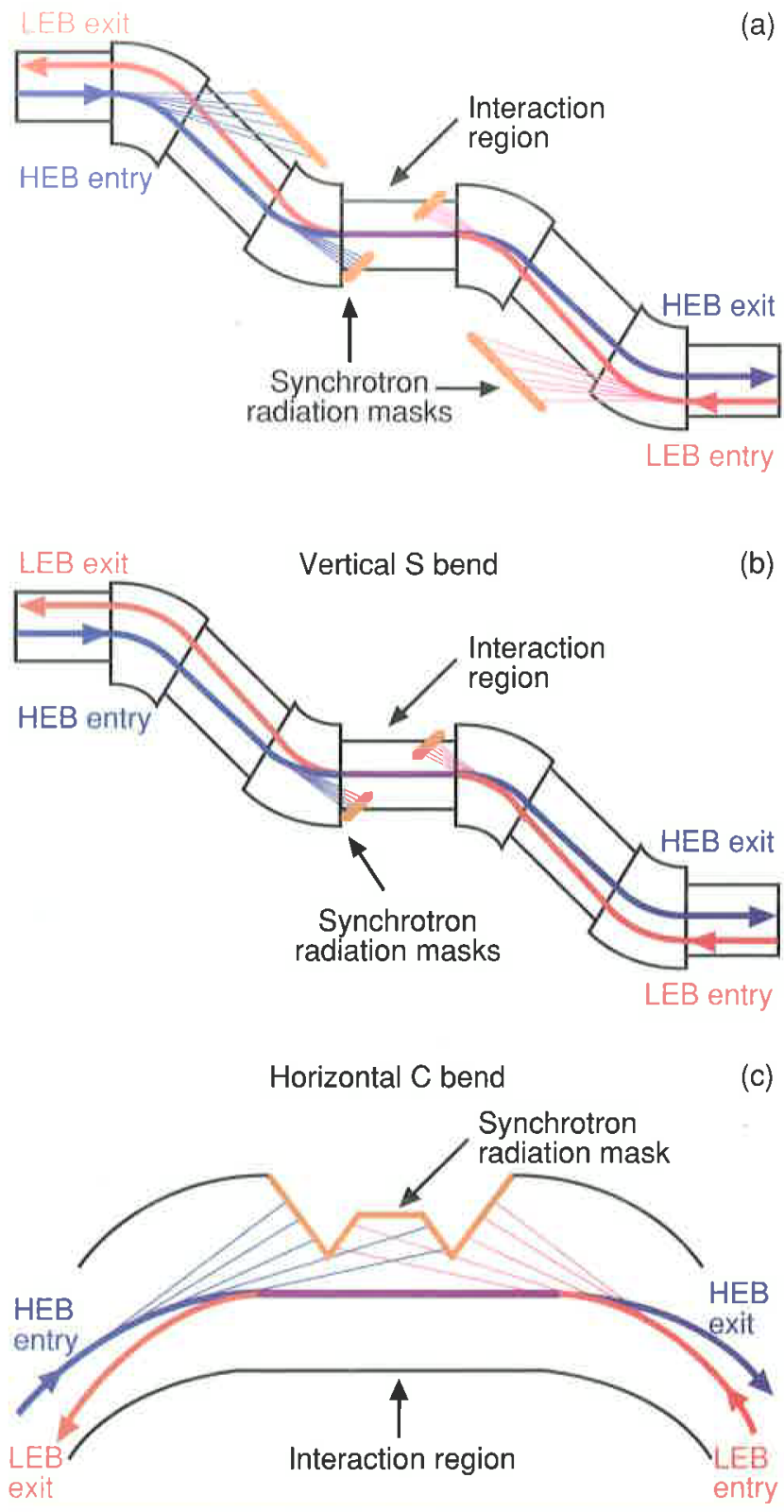

FICURE 3

Design of the interaction region of a collider, still in a state of flux, with a number of interesting ideas being considered, but with no consensus as to how best to proceed. One possibility is an S-bend, head-on configuration, which is shown in (a). This appears to be good for masking of the detector, while allowing for subsequent modification so as to have crossing at an angle. Other ideas include a configuration where the high-energy beam goes through the centres of focusing quadrupoles, use of combined function magnets, and "tilting" of the detector solenoid so as to facilitate beam separation. In (b) we show a three-dimensional bend ( $S$ vertically) and in (c) ( $C$ horizontally), a "propeller blade" crossing which might be quite advantageous as far as masking is concerned. sect. 5) and it appears to be more difficult to design an interaction region with a round beam (but small cross section) rather than with a flat beam (very small vertically, but big horizontally), thus the obvious advantage of a factor of two, in round beams versus flat beams, is washed out. Also in favour of flat beams, there appears to be less synchrotron radiation in that case because the required focusing gradients are lower than those needed to produce round beams. Presently, there is no unanimity of thought on the subject of round vs flat beams.

Still another aspect of the interaction region is whether or not the collisions are head-on or crossing at an angle. Certainly a non-zero crossing angle reduces the masking problem greatly, but crab crossing, which would be necessary, has not yet been tried. In fig. 4 we indicate the nature of crab crossing. The luminosity of a head-on configuration would be maintained in the crossing case but, much more importantly, the transverse beam-beam kick does not couple to the longitudinal degree of freedom of the particles, and hence the beam-beam interaction is no different in the crossing case than in the head-on case. Study and simulations of the effects of crab crossing, in synergism with beam-beam effects, is just starting. Most projects are not "counting on crab crossing", but are allowing for the possibility of incorporating this feature in the future (i.e. by having $S$ bends in the case of head-on collisions)

\section{Single-beam phenomena}

The subject of single-beam instabilities has been well-studied through the years in connection with storage rings and colliders. The new synchrotron radiation sources are being built with very short bunches (so as to get good time resolution of the radiation) and with very small emittance bunches (so as to have very bright sources). Their construction has been based upon our knowledge and experience with colliders, but the frontiers of research on single-beam instabilities are now being pushed by the people concerned with synchrotron radiation sources [6].

A comprehensive discussion of intense beam phenomena can be found in many laboratory design study reports and, in particular, in two recent papers ([7] and [8]). One must consider the longitudinal microwave instability, transverse modecoupling instability, and coupled-bunch instabilities. It is the last that are the most serious. They are driven by the impedance of the RF cavities and for the regime of total current under consideration for a $B$ Factory, have growth times for the worst modes of the order of a millisecond (recall that synchrotron radiation demands RF cavities with power in the $10 \mathrm{MW}$ range). Such rapid instabilities must be controlled by very powerful feedback systems; that is, systems of wide bandwidth and having considerable amplifier power. It is not novel to employ such systems (they are presently used on a number of machines) but the present demands on power and bandwidth are in excess of current practice. 


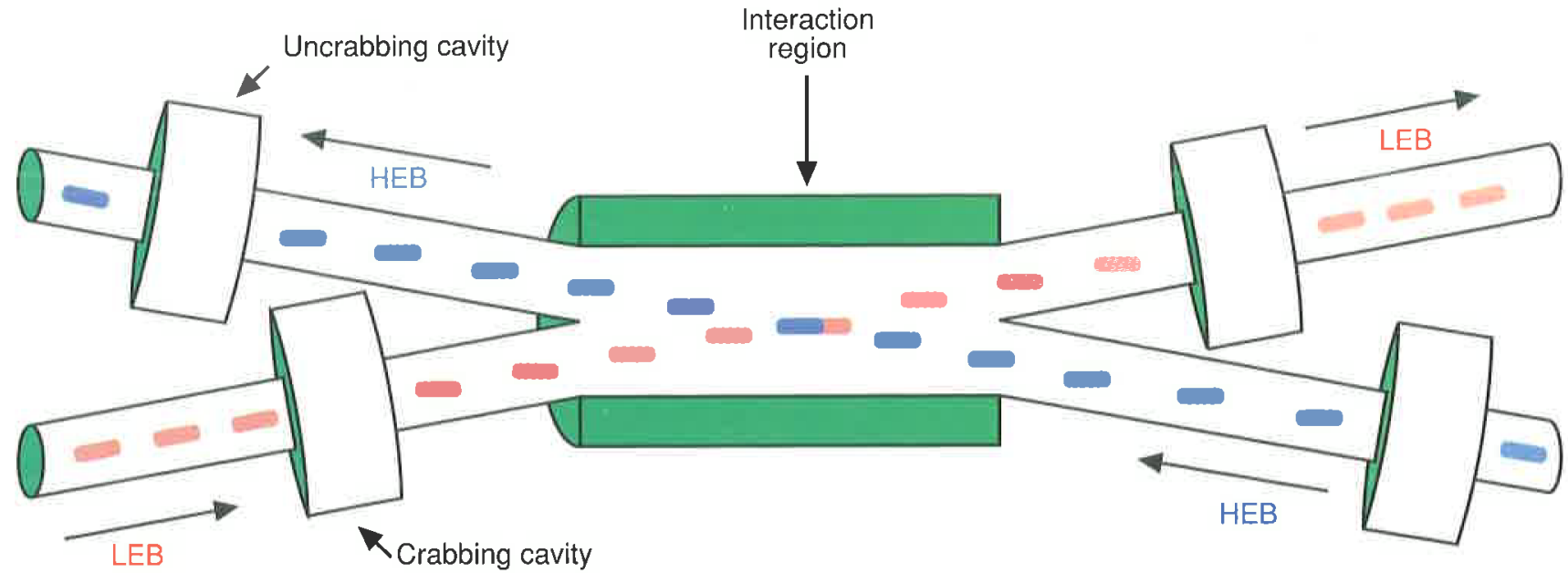

FIGURE 4

A diagram of "crab crossing" which shows how by tilting the bunches (by half the crossing angle, which typically is $\sim 25$ mrad) the crossing appears "head-on" in a moving frame (up in the diagram). Notice that one needs to tilt the bunches and then un-tilt them after the crossing. Powerful RF cavities are required to do the necessary gymnastics and they have to be reasonably close to the interaction point and carefuliy adjusted to avoid introducing synchro-betatron resonances.

Because coupled-bunch instabilities need to be reduced as much as possible, there is the need to reduce the impedances of the higher modes in the RF cavities as much as possible. This can be accomplished by making the cavity bore large, damping the higher-order modes, and using as few cavities as possible (i.e. operate at a high gradient). The last demands the ability of "windows" to transmit great RF power, and that requires new technology. The issue of room temperature or superconducting cavities is not yet settled. Notice that the crab cavities (which will give increased impedance, and therefore are a negative element in the crab crossing scheme) will likely be superconducting cavities as they demand voltage, but do not demand power.

Finally, we should mention other single-beam phenomena that are not limiting, but need to be considered in the design. These include radiation damping, quantum excitation, intra-beam scattering, Touschek scattering and gas scattering. For example, consideration is being given to whether the vacuum chamber should be made of aluminium and have an antechamber to absorb the synchrotron heating, or whether it is allowable to have a single chamber made of copper.

Of more than passing interest is the collection of ions in the electron beam. This matter is well known, but still not entirely understood. The clearing of unwanted ions (without introducing excessive impedance from the clearing electrodes, which will drive various instabilities or without losing luminosity, as will be the case if one imposes a long gap in the train of bunches) is possible, but not easy.

\section{Two-beam phenomena}

The beam-beam interaction is the heart of any collider. But the beam-beam coherent electromagnetic interaction - a particle of one beam interacting with the total electric and magnetic fields of the other beam - is an unwanted component of the collision and, very importantly, puts a severe limit on the operation of the collider. The beam-beam interaction has been studied, both theoretically and experimentally, for decades [9]. This effect is often treated in the "weak-strong" approximation, which consists of one particle interacting with a prescribed intense beam. A proper analysis must, however, include strongstrong phenomena such as coherent beam-beam effects and instabilities.

The beam-beam effect is usually quantified in terms of the linear lens effect of one beam on the other. It is clear, of course, that any linear effect can be compensated and that it is really the non-linear part of the interaction (which is proportional to the linear lens effect) that is important. Luminosity $L$ of a collider is given by

$$
L=\frac{N^{+} N^{-} f k}{4 \pi \sigma_{\mathrm{x}} \sigma_{\mathrm{y}}},
$$

where $N^{+}, N^{-}$are the bunch particle numbers, $f$ is the frequency of rotation, $k$ is the number of bunches in the collider, and $\sigma_{\mathrm{x}}, \sigma_{\mathrm{y}}$ are the horizontal and vertical beam sizes, respectively (assumed the same in the two rings). The vertical beam-beam strength parameter $\xi_{\mathrm{y}}$ is given by 


$$
\xi_{\mathrm{y}}^{ \pm}=\frac{N^{\mp_{l_{\mathrm{c}}} \beta_{\mathrm{y}} \pm}}{2 \pi \gamma^{ \pm}\left(\sigma_{\mathrm{x}}+\sigma_{\mathrm{y}}\right) \sigma_{\mathrm{y}}} .
$$

where $r_{\mathrm{e}}$ is the classical electron radius, $\gamma$ is the energy of the beam in units of rest mass energy, and the $\beta^{*}$ value is introduced explicitly.

Combining these formulas we arrive at

$$
L=\frac{f k(1+r)}{2 r_{\mathrm{c}}}\left[\frac{N^{+} \gamma^{+} \xi_{\mathrm{y}}^{+}}{\beta_{\mathrm{y}}^{++}}\right]^{1 / 2}\left[\frac{N^{-} \gamma^{-} \xi_{\mathrm{y}}^{-}}{\beta_{y}^{+1 / 2}-}\right]^{1 / 2} .
$$

where $r$ is the aspect ratio of the beams ( 1 for round, 0 for flat). In deriving this formula it has been assumed that the beam-beam interaction in the vertical direction is the limiting phenomenon. The beam-beam strength parameter $\xi$, both experimentally and theoretically, is within the range 0.03 to 0.06 . Thus, we see that high luminosity requires high beam current and low $\beta^{*}$ (and that these two quantities can be varied arbitrarily provided the beam size is properly adjusted). There are, of course, other limits on the low $-\beta^{*}$ value and the beam current.

At first sight, it appears that round beams are better than flat beams (by a factor of two), and this effect may be even greater than is explicit if $\xi$ depends on the beam aspect ratio. At present, the dependence of $\xi$ on aspect ratio is moot. It appears to be more difficult to make a low- $\beta^{*}$ lattice for round beams than for flat beams, by about a factor of two, which removes the obvious advantage of round beams. Thus, it is unclear at this time whether round beams offer any advantage over flat beams.

The beam-beam interaction will be more severe if the bunch is comparable to, or longer than, the $\beta^{*}$ at the crossing point. This is because $\beta^{*}$ increases quickly (quadratically with distance) as one moves away from the crossing point. Thus, it is necessary to have short bunches, which requires lots of RF voltage. In fact, the necessary length of bunches precludes making $\beta^{*}$ very small (and hence limits the amount of luminosity possible with a single pair of bunches).

The beam-beam interaction tends to throw particles out to large amplitudes and this results in short-beam lifetime and aggravated detector background. Radiation damping has the opposite effect and it is true that a collider performs better when the radiation damping is large. Just how much damping is required for various operating conditions is not yet clear. It is a matter under study at this time.

The beam-beam interaction can also lead to motion of the beam as a whole (rather than the incoherent effect discussed above). It is important to avoid coherent instabilities, and that appears possible in practice. Finally, then, all projects are not considering moving into a new ground with the beam-beam interaction (except in having $\beta^{*}$ very small, i.e. of the order of the bunch length), but plan on obtaining the improved luminosity over present colliders by means of having many bunches.

\section{Projects}

There is great interest, throughout the world, in the development of a $B$ Factory. Serious design studies are now under way at six different institutions; namely, Cornell in Ithaca |10|, KEK in Tsukuba |11|, INP in Novosibirsk [12|, CERN in Geneva (in collaboration with the Paul Scherrer Institute) [13], DESY in Hamburg |14|, and SLAC/LBL in Stanford [15]. Four of the projects are based on existing rings; namely PEP at SLAC, the ISR at CERN, CESR at Cornell, and PETRA at DESY. In addition, there are studies, al CERN | 16] and at CEBAF |17], of a Linac colliding with a ring

The projects are still in a very preliminary state, with some of them hoping to have a reasonably firm parameter list before the end of the year. It appears at present that there is a convergence of design parameters (Linac options aside) so that there is considerable similarity among the various projects (a year ago, one could not have said that). To illustrate the range of parameters under study, we show in table 1 the present design parameters of three of these projects. It seems likely that many of the parameters will change before the projects become actual proposals. The SLAC/LBL parameters are for round beams, but that group is now developing a flat-beam case, which may be what it actually proposes. The Cornell group plans to start with a symmetric collider and then go to an asymmetric case (the asymmetric case is the one listed). The Novosibirsk beams have correlated dispersions at the interaction point that result in a "narrowing" of c.m. energy spread. This may be desirable from an experimental point of view, but may worsen the beam-beam effect. The last is being studied right now, with initial results looking encouraging.

In conclusion, the construction of a $B$ Factory to study $B$ meson physics and CP violation in that system seems, from the beam physics point of view, to be feasible, but challenging. Feasibility studies are now under way to quantify the challenge.

\section{Acknowledgements}

Thanks are given to the attendees at the Workshop on HighLuminosity Asymmetric Collider Rings, Lawrence Berkeley Laboratory, 12-16 February 1990. I wish to thank Gil Travish for making figs 3 and 4 . I also wish to thank S. Chattopadhyay, G. Lambertson, M. Tigner and M. Zisman for a careful reading of the paper and a number of useful suggestions. This work was supported by the Director, Office of Energy Research, Office of High-Energy and Nuclear Physics, Division of High-Energy Physics, of the US Department of Energy under contract DE-AC03-76SF00098. 
Table 1: Typical parameters of $B$ Factory projects

\begin{tabular}{|c|c|c|c|c|c|c|}
\hline & \multicolumn{2}{|c|}{ SLAC/LBL } & \multicolumn{2}{|c|}{ Novosibirsk } & \multicolumn{2}{|c|}{ Cornell } \\
\hline & $\begin{array}{c}\text { Low-energy } \\
\text { ring }\end{array}$ & $\begin{array}{l}\text { High-energy } \\
\text { ring }\end{array}$ & $\begin{array}{c}\text { Low-energy } \\
\text { ring }\end{array}$ & $\begin{array}{c}\text { High-energy } \\
\text { ring }\end{array}$ & $\begin{array}{c}\text { Low-energy } \\
\text { ring }\end{array}$ & $\begin{array}{l}\text { High-energy } \\
\text { ring }\end{array}$ \\
\hline Energy, $E[\mathrm{GeV}]$ & 3.1 & 9 & 4.3 & 6.5 & 3.5 & 8.0 \\
\hline Circumference, $C \mid \mathrm{m}]$ & 2200 & 2200 & 655 & 655 & 768 & 768 \\
\hline Number of bunches, $k_{\mathrm{B}}$ & 1296 & 1296 & 156 & 156 & 96 & 96 \\
\hline Particles per bunch, $N_{\mathrm{b}}\left[10^{10}\right]$ & 7.88 & 5.44 & 9 & 6 & 37 & 16 \\
\hline Total current, $I[\mathrm{~A}]$ & 2.23 & 1.54 & 1 & 0.7 & 2.19 & 0.96 \\
\hline \multicolumn{7}{|l|}{ Emittance } \\
\hline$\varepsilon_{\mathrm{x}}[\mathrm{nm} \mathrm{rad}]$ & 66 & 33 & 8 & 6.5 & 78 & 78 \\
\hline$\varepsilon_{\mathrm{y}}[\mathrm{nm} \mathrm{rad}]$ & 66 & 33 & 0.25 & 0.25 & 78 & 78 \\
\hline Bunch length, $\sigma_{\mathrm{I}}[\mathrm{mm}]$ & 10 & 10 & 7.5 & 7.5 & 18 & 18 \\
\hline Momentum spread, $\left.\sigma_{\mathrm{p}} \mid 10^{-4}\right]$ & 9.5 & 6.1 & 10 & 10 & 3.6 & 8.4 \\
\hline \multicolumn{7}{|l|}{ Damping time } \\
\hline$\tau_{x, y}[\mathrm{~ms}]$ & 32.3 & 37 & 17 & 13 & 26 & 26 \\
\hline$\tau_{\mathrm{E}}[\mathrm{ms}]$ & 17.3 & 18.5 & - & - & 13 & 13 \\
\hline \multicolumn{7}{|l|}{ Beta functions at $I P$} \\
\hline$\beta_{\mathrm{x}}^{*}[\mathrm{~cm}]$ & 3 & 6 & 60 & 60 & 3 & 3 \\
\hline$\beta_{y}^{*}[\mathrm{~cm}]$ & 3 & 6 & 1 & 1 & 3 & 3 \\
\hline \multicolumn{7}{|l|}{ Betatron tune } \\
\hline horizontal, $v_{\mathrm{x}}$ & 37.76 & 21.28 & 29 & 26 & 7.04 & 10.7 \\
\hline vertical, $v_{y}$ & 35.79 & 18.20 & 20 & 13 & 7.04 & 10.7 \\
\hline Synchrotron tune, $Q_{\mathrm{s}}$ & 0.039 & 0.053 & 0.028 & 0.028 & 0.05 & 0.05 \\
\hline Momentum compaction, $\alpha$ & 0.00115 & 0.00245 & 0.002 & 0.002 & 2.02 & 0.88 \\
\hline \multicolumn{7}{|l|}{ RF parameters } \\
\hline frequency, $f_{\mathrm{rf}}[\mathrm{MHz}]$ & 353.2 & 353.2 & 500 & 500 & 500 & 500 \\
\hline voltage, $V_{\mathrm{rf}}[\mathrm{MV}]$ & 10 & 25 & 8.8 & 15 & 2.2 & 11.3 \\
\hline \multicolumn{7}{|l|}{ Nominal beam-beam tune shift } \\
\hline$\xi_{\mathrm{ox}}$ & 0.03 & 0.03 & 0.012 & 0.012 & 0.045 & 0.045 \\
\hline$\xi_{\text {oy }}$ & 0.03 & 0.03 & 0.05 & 0.05 & 0.045 & 0.045 \\
\hline Luminosity, $L\left[\mathrm{~cm}^{-2} \mathrm{~s}^{-1}\right]$ & & $10^{33}$ & & $10^{33}$ & & $10^{33}$ \\
\hline
\end{tabular}

\section{References}

[1] Linear Collider $B \bar{B}$ Factory Conceptual Design, editor D.H. Stock, World Scientific (1987);

The physics program of a high-luminosity asymmetric $B$ Factory, SLAC 353, LBL/PUB-5245, CALT-68-1588 (1989);

J. Dorfan, to be published in Particle World (1990).

[2] Workshop on Beam Dynamics Issues of High-Luminosity Asymmetric Collider Rings, American Inst. of Phys. Conf. Proc. (1990) to be published.

13] The physics program of a high-luminosity asymmetric $B$ Factory, SLAC-353, LBL PUB-5245, CALT-68-1588 (1989).
14] R.B. Palmer, Energy scaling, crab crossing and the pair problem, SLAC-PUB-4707 (1988);

$\mathrm{K}$. Oide and K. Yokoya, Crab crossing scheme for storage ring colliders, SLAC-PUB-4832 (1989);

K. Oide, Asymmetric collider with crossing angle, Workshop on High-Luminosity Asymmetric Rings for $B$ Physics, CalTech, CALT-68-1552 (1989).

[5] M. Sands, The physics of electron storage rings - An introduction, Stanford Linear Accelerator Center Report No. SLAC-121, National Technical Information Service, Springfield, Virginia, USA (1970). 


\section{References (cont'd)}

[6] M.S. Zisman, Influence of collective effects on the performance of high-brightness synchrotron radiation sources, Proc. of JAERI-Riken Symposium on Accelerator Technology for the High-Brilliance Synchrotron Radiation Sources, Tokyo, Japan (1988) 311-346;

S. Chattopadhyay, Stability of high brilliance synchrotron radiation sources, 6th National Conf. on Synchrotron Radiation Instrumentation, Berkeley, USA (1989), to be published in Nucl. Instr. and Meth. A;

A. Jackson, The challenges of third-generation synchrotron light sources, Proc. of the XIVth Intern. Conf. on HighEnergy Accelerators, Tsukuba, Japan (1989), to be published in Particle Accelerators.

[7] R.H. Siemann, $B$ Factories: A prospective of $B$ physics and possible accelerator design approaches, Proc. of the 1988 Linear Accelerator Conf., Newport News (1988).

[8] R.H. Siemann, The accelerator challenges of $B$ Factories, Proc. of the XIVth Intern. Conf. on High-Energy Accelerators, Tsukuba, Japan (1989), to be published in Particle Accelerators.

[9] E. Keil, Beam-beam effects in electron and proton colliders, Proc. of the 1989 IEEE Particle Accel. Conf., IEEE-89CH2669-0 (1990) 1731.

[10] K. Berkelman, invited talk presented at the La Thuile Symposium, 29 February to 5 March 1988;

M. Tigner, Workshop on Beam Dynamics Issues of HighLuminosity Asymmetric Collider Rings, American Inst. of Phys. Conf. Proc. (1990), to be published.

[11] F. Abe et al., Proposal for study of $B$ physics by a detector with particle identification and high-resolution calorimetry at the TRISTAN accumulator ring, KEK Report (1988).

[12] A.N. Dubrovin, A.N. Skrinsky, G.N. Tumaiki and A.A. Zholents, Conceptual design of a ring beauty Factory, EPAC Accelerator Conf., Rome, Italy, 1 (1988) 467;

A.A. Zholents, Workshop on Beam Dynamics Issues of High-Luminosity Asymmetric Collider Rings, American Inst. of Phys. Conf. Proc. (1990), to be published.
[13] Proposal for an electron-positron collider for heavy flavor particle physics and synchrotron radiation, report from Paul Scherrer Inst., Villigen, Switzerland PR-88-09 (1988).

[14] H. Nesemann, W. Schmidt-Parzefall and F. Willeke, The use of PETRA as a $B$ Factory, EPAC Accel. Conf., Rome, Italy, 1 (1988) 439.

[15] Feasibility study for an asymmetric $B$ Factory based on PEP, LBL Pub-5244/SLAC-3521, CALT-68-1589 (1989); Investigation of an asymmetric $B$ Factory in the PEP tunnel, LBL Pub. 5263/SLAC-359/CALT-68-1622 (March 1990).

[16] P. Grosse-Wiessmann et al, CERN Linac-on-ring option, Workshop on Beam Dynamics Issues of High-Luminosity Asymmetric Collider Rings, American Inst. of Phys. Conf. Proc. (1990), to be published.

[17] S. Heifets, The CEBAF $B$ Factory project, Workshop on Beam Dynamics Issues of High-Luminosity Asymmetric Collider Rings, American Inst. of Phys. Conf. Proc. (1990), to be published.

Address:

Andrew M. Sessler

Lawrence Berkeley Laboratory

University of California

USA-Berkeley, CA 94720

Received by A. Poskanzer, May 1990. 